\title{
AVALIAÇÃO DA POPULAÇÃO DE NEMATÓIDES EM BANANAL COM E SEM O USO DE ORGANOMINERAL ${ }^{1}$
}

\author{
CECÍLIA HELENA SILVINO PRATA RITZINGER ${ }^{2}$, MARILENE FANCELLI ${ }^{3}$, \\ ZILTON JOSÉ MACIEL CORDEIRO ${ }^{3}$, ROSIANE DA SILVA VIEIRA ${ }^{4}$, \\ CARLOS ALBERTO DA SILVA LEDO ${ }^{3}$
}

RESUMO - Com a demanda pela produção integrada, o monitoramento torna-se fundamental para reduzir ou substituir insumos poluentes na fertilização e no manejo de pragas. Muitos produtos têm sido aplicados em áreas de produção, sem o devido registro ou conhecimento de sua eficiência ou de seu efeito sobre pragas e inimigos naturais. Nesta região, o uso de organomineral é preconizado como alternativa ao uso de fertilizante e possivelmente com efeito nematicida. As avaliações, para verificar o efeito deste produto, foram realizadas, por meio de cinco coletas, no período de 2008 a 2010, em três propriedades, em bananais, cv. 'Williams', introduzidos há oito e 13 anos, dentro do projeto de Irrigação de Formoso, em Bom Jesus da Lapa-BA. Foram coletadas dez amostras compostas de duas subamostras de solo e raízes, em área com e sem a aplicação de organomineral. As propriedades, três, foram denominadas, área 1, 2 e 3 . A analise de variância foi feita considerando: três épocas de avaliação, dois tratamentos (com e sem organomineral) e três propriedades). O nível de dano foi determinado por meio de escala visual e os dados submetidos à ANOVA. Houve interação significativa entre populações de nematoide no ano e propriedade e entre organomineral e propriedade $(\mathrm{P} \leq 0,01)$. Não houve correlação entre nível de dano e organomineral nas áreas 1 e 3. Contudo, houve correlação negativa entre o nível de dano e nematoides na presença de organomineral, na área $3(\mathrm{P} \leq 0,01)$. Meloidogyne spp. foram as espécies que ocorreram em maior população em Bom Jesus da Lapa-BA. A utilização do organomineral não proporcionou redução significativa na população de nematoides. O nível de dano foi baixo, não podendo ser explicado pelo uso do organomineral. O manejo cultural interferiu no desenvolvimento da população de fitonematoides e de nematoides de vida livre.

Termos para indexação: Produção integrada, Musa spp., monitoramento.

\section{EVALUATION OF THE NEMATODE POPULATION IN BANANA PLANTATIONS WITH AND WITHOUT THE USE OF ORGANOMINERAL}

\begin{abstract}
With the demand for integrated production, monitoring becomes fundamental to reduce or to substitute pollutant inputs in the fertilization and in the management of pests. Many products have been used in production areas, without the proper registration or knowledge of its efficiency or its effect on pests and natural enemies. In the studied area, the use of organomineral is recommended as an alternative to the use of fertilizers and possibly with nematicidal effect. The evaluations, to verify the effect of this product, were accomplished, through five samplings, in the period of 2008 to 2010, in three properties, cultivated with banana cv. 'Williams' with the age ranging from 8 months to 13 years, in the Irrigation Project of Formoso, in Bom Jesus da Lapa, BA. In the study, ten samples composed by two subsamples of soil and roots were collected, in areas with and without the organomineral application. The damage level was determined using the visual scale and the data was submitted to ANOVA. There was significant interaction of nematode populations between years and properties and between organomineral utilization and properties $(\mathrm{P} \leq 0.01)$. There was no correlation between damage level and organomineral utilization in the areas 1 and 3. However, there was negative correlation between the damage level and nematodes in the organomineral presence, in the area $3(\mathrm{P} \leq .01)$. Meloidogyne spp. was the species that had the highest population in Bom Jesus da Lapa, BA. The use of organomineral did not provide significant reduction in the nematode population. The damage level was low, which could not be explained by the use of the organomineral. Cultural practices interfere in the development of the plant nematode population and free life nematodes.
\end{abstract}

Index terms: Integrated production, Musa spp., monitoring.

\footnotetext{
1'(Trabalho 002-11). Recebido em: 03-01-2011. Aceito para publicação em: 24-08-2011. Apoio financeiro: MAPA/CNPq ${ }^{2}$ Eng. Agr., PhD., Embrapa Mandioca e Fruticultura Tropical, C.P. 007, 44380-000, Cruz das Almas-BA. E-mail: cecilia@cnpmf.embrapa.br ${ }^{3}$ Eng. Agr., D.Sc., Embrapa Mandioca e Fruticultura Tropical, C.P. 007, 44380-000, Cruz das Almas-BA. E-mails: fancelli@cnpmf.embrapa.br; zilton@cnpmf.embrapa.br; ledo@cnpmf.embrapa.br

${ }^{4}$ Mestranda, Centro de Ciências Agrárias, Ambientais e Biológicas, Universidade Federal do Recôncavo da Bahia, Rua Rui Barbosa, 710 - Centro, 44380-000, Cruz das Almas-BA. E-mail: anesvieira@yahoo.com.br. Ex-bolsista da FAPESB.
} 


\section{INTRODUÇÃO}

A bananicultura é uma atividade de grande importância econômica e social para o Brasil, que produz cerca de sete milhões de toneladas anuais (FAO, 2010). Os fitonematoides constituem uma das principais limitações à cultura, sendo responsáveis por $20 \%$ de queda na produção, chegando a $50 \%$ em algumas situações. Dentre os fitonematoides, são destacados Radopholus similis, Helicotylenchus multicinctus, Pratylenchus spp. (GAIOSWISK, et al., 2009; HARTMAN et al., 2010).

Vários são os perímetros irrigados onde a bananicultura se destaca. Em áreas de produtores situados no projeto de Irrigação do Formoso, em Bom Jesus da Lapa-BA, foi identificado sério problema causado pelo nematoide das galhas (Meloidogyne spp.), com perdas na produção. Esse nematoide assume importância econômica devido à dificuldade de controle e facilidade de dispersão, por meio dos tratos culturais (RITZINGER et al., 2008).

A produção integrada (PI) é um sistema que emprega tecnologias que requerem a aplicação de Boas Práticas Agrícolas (BPA), buscando a garantia de mercado, mediante a prática de um sistema de cultivo economicamente rentável, ambientalmente sustentável e socialmente justo (PORTOCARRERO; KOSOSKI, 2009). É importante salientar que o uso de praguicidas não é proibido na PI, porém esses produtos devem ser registrados no MAPA para uso na cultura e aprovados/citados na grade de agroquímicos (CORDEIRO et al., 2009). Nas propriedades trabalhadas, que estavam adequandose às normas da produção integrada, dois sistemas eram utilizados para a fertilização dos bananais (com e sem biofertilizantes), sendo observado pelos produtores um possível efeito praguicida. Entretanto, esses produtos não apresentam registro ou conhecimento de sua eficiência contra pragas e inimigos naturais. Dessa forma, é relevante que a experiência do produtor seja validada pela pesquisa, propiciando importantes informações para ajustes das normas da PI, buscando o melhor ajuste no sistema de produção.

O objetivo deste trabalho é identificar e quantificar a ocorrência de fitonematoides e nematoides de vida livre e avaliar o nível de dano em bananais com e sem aplicação de produto organomineral.

\section{MATERIAL E MÉTODOS}

O trabalho foi realizado no período de abril de 2008 a junho de 2010. Foram selecionadas três áreas produtoras de banana, designadas como propriedades 1; 2 e 3, localizadas no projeto de Irrigação do Formoso, em Bom Jesus da Lapa-BA, as quais utilizavam um produto organomineral como biofertilizante. As áreas cultivadas com a bananeira 'Williams' no $2^{\circ}$ ciclo foram demarcadas para instalação dos experimentos com e sem a utilização do produto. Em cada propriedade, foram georreferenciados quatro pontos delimitando a área para as amostragens nas parcelas com e sem organomineral. A composição do fertilizante organomineral, de acordo com o fabricante, é: pasta húmica, biocatalisador $\mathrm{N} 25, \mathrm{KCl}$, ácido bórico, sulfato de magnésio, sulfato de zinco, MAP, biosulfre, micronutrientes e ácido fúlvico.

Na propriedade 1, a área foi plantada no espaçamento de 4,0 m x (2,0 m x 2,0 m). A adubação foi feita mensalmente, apenas com fontes minerais e de acordo com análise do solo, inclusive na área submetida à aplicação do organomineral. Selecionaram-se oito fileiras duplas de 15 plantas cada, perfazendo-se um total de 240 plantas para cada parcela.

Na propriedade 2, as observações foram feitas em bananal sob solo arenoso, no espaçamento $4,0 \mathrm{~m} \times(2,0 \mathrm{~m} \times 1,8 \mathrm{~m})$, perfazendo um total de 200 plantas para cada parcela. Registraram-se aplicação de carbofuran e intenso ataque de Cosmopolites sordidus.

Na propriedade 3, a aplicação do organomineral foi feita em toda a área, por aproximadamente três anos. Nesta área, o solo é argiloso, e o espaçamento utilizado é de 4,0 $\mathrm{m}$ x (2,0 $\mathrm{m}$ x 1,8 m). Foram marcadas quatro fileiras duplas de 25 plantas cada, perfazendo-se o total de 200 plantas. As observações em vermelho estão corretas. Colocamos entre parentes nessa última para uniformizar).

Para quantificar a população inicial (Pi) de fitonematoides e nematoides de vida livre, em abril de 2008, procedeu-se à amostragem no solo por meio de duas amostras simples por planta, totalizando-se 10 amostras por área. As amostras foram retiradas em lados opostos da bananeira-mãe, no sentido de condução do bananal. As demais amostras foram retiradas em setembro de 2008 , fevereiro e agosto de 2009 e junho de 2010. A extração de nematoides foi feita no Laboratório de Nematologia da Embrapa Mandioca e Fruticultura Tropical, adotando-se o método descrito por Jenkins (1964). O nível de dano nas raízes foi determinado utilizan- 
do-se a escala visual, conforme descrita em metodologias para monitoramento por Cordeiro e Fancelli (2008), considerando-se amostras referentes a 2009 e 2010. Na ocorrência de Meloidogyne, os isolados foram identificados no Laboratório de Nematologia de Petrolina-PE.

As plantas amostradas tinham o cacho já emitido e a planta-filha com, aproximadamente, $1,50 \mathrm{~m}$ de altura, conforme descrito em metodologia para monitoramento (CORDEIRO; FANCELLI, 2008). Essa padronização, dentro da mesma área, evitou a amostragem em plantas com formação de cacho e plantas com cacho em fase de colheita, bem como em plantas que ainda não tinham lançado cacho, pois essa variabilidade pode afetar a preferência do nematoide devido à diferença de raízes ativas.

Os dados obtidos foram submetidos à análise de variância considerando o delineamento inteiramente casualizado, no esquema fatorial $3 \times 2 \times$ 3 (três épocas de avaliação, dois tratamentos e três propriedades). As médias dos tratamentos foram comparadas pelo teste de Tukey, a 5\% de probabilidade.

\section{RESULTADOS E DISCUSSÃO}

Foram identificadas populações de Meloidogyne incognita e M. sp., Helicotylenchus sp., Criconemella sp., Rotylenchulus reniformis, Hoplolaimus sp. e Pratylenchus sp.. Contudo, devido à baixa população e grande variabilidade das populações de Helicotylenchus sp., Criconemella sp., Rotylenchulus reniformis, Hoplolaimus sp. e Pratylenchus sp., nas três propriedades, sendo discutidos somente os resultados para as populações de Meloidogyne spp. e nematoides de vida livre, que foram mais constantes e representativos nas três localidades, em todas as avaliações. Como não podem ser quantificadas as espécies de Meloidogyne no solo, os resultados são discutidos, utilizando-se de Meloidogyne spp..

Houve interação significativa $(\mathrm{P} \leq 0,01)$ entre ano e localidade, e entre organomineral e localidade, $(\mathrm{P} \leq 0,05)$ apenas para Meloidogyne spp. (Tabela 1).

Houve tendência de diminuição da população de Meloidogyne e aumento de nematoides de vida livre nas áreas 1 e 3 . Na área 2, houve aumento acentuado da população de Meloidogyne com redução drástica em 2010. Nesta área, constatou-se baixa população de nematoides de vida livre, possivelmente, devido ao uso de pesticidas, conforme relato do produtor (Figuras 1 e 2).

De maneira geral, observou-se diferença para o manejo adotado e estado nutricional das plantas entre as áreas amostradas no Projeto For- moso (dados não apresentados). Na Propriedade 1, observou-se maior acompanhamento de práticas culturais, menor uso de produtos químicos e uso de análises do solo para aplicação do organomineral e, consequentemente, menor variabilidade nas parcelas tratadas com e sem organomineral, tendo em vista que as demais práticas foram as mesmas nas duas parcelas.

Houve correlação negativa entre a população de Meloidogyne spp. no solo e o nível de dano na localidade $2 \mathrm{com}$ organomineral (Tabela 1). Todavia, verifica-se que, embora a maior porcentagem de raízes apresentasse o nível de dano nas escalas 0 e 1, que correspondem a até $25 \%$ de dano (Figura 3). Essa avaliação foi feita somente em raízes vivas, não se considerando as raízes mortas.

Os parâmetros avaliados, densidade populacional e nível de dano, estão mais relacionados ao manejo adotado em cada propriedade, tendo em vista que as condições entre uma propriedade e outra foram distintas, do que, propriamente, ao organomineral preconizado como fertilizante e/ou nematicida. Trabalhos desenvolvidos por Gaidashova et al. (2009) e Hartman et al. (2010) revelam que, em avaliações de danos por nematoides, devem ser considerados também outros fatores que resultam em necrose das raízes. Na avaliação de nível de dano, não foram computadas as raízes mortas e, por ocasião da primeira amostragem, em abril de 2008, a população inicial de nematoides na área antes da aplicação do organomineral não era conhecida. Assim, a correlação negativa entre a população de nematoides no solo e o nível de danos na propriedade 2, sob organomineral, pode ser atribuída à pressão da população inicial de nematoides e às condições de manejo de cada propriedade. Há possibilidade, inclusive, de diferentes manejos (incluindo frequência de adubações, controle de pragas e tratos culturais) nas três propriedades terem interferido no nível de dano. Esses resultados corroboram os com experimentos conduzidos por Gaidashova et al. (2009); por essa razão, sugere-se a realização de experimentos sob condições controladas para definir níveis de dano por nematoides, haja vista as inúmeras interações envolvendo populações de nematoides, que ocorrem num bananal. Sabe-se que a ocorrência de ventos ou problemas nas tubulações de irrigação ou fertilização ocorreram em alguns setores, amostrados nas diferentes propriedades, promovendo, inclusive, atraso na condução dos tratos culturais como eliminação de perfilho ou mesmo na data de aplicação do organomineral. Trabalhos desenvolvidos por Gaidashova et al. (2009) revelam que, independentemente do tipo de solo, 
bananais, mesmo sob altas populações $P$. goddey, podem ter seus danos mascaradas, pelo alto vigor da planta, favorecido pela densidade de plantas e práticas de cobertura.

Como destacado, o manejo pode ter exercido maior influência no nível de dano por nematoide do que o organomineral. Vale ressaltar que o nematoide das galhas é mais favorecido em solos arenosos do que nos argilosos. Por isso, considerando a análise granulométrica, porosidade total, macro e microporosidade e curva de retenção de água, segundo trabalhos desenvolvidos por Borges e Souza (2009), solos variando de arenosos a argilosos não devem receber o mesmo manejo de irrigação, como vem acontecendo naquela região. Certamente, os problemas registrados naquela região em relação à aplicação de água, seja excessiva em algumas localidades, seja deficiente em outras, promovem aeração deficiente, apodrecimento de raízes, lixiviação de nutrientes para fora da zona radicular, tombamento de plantas, acontecimentos que prejudicam a produção* de banana. Assim, a ocorrência de nematoides nos bananais pode ser amplificada pelo manejo inadequado de irrigação*. $\mathrm{O}$ uso correto da irrigação deve ser considerado não só de acordo com a condução do bananal, mas também com o tipo de solo e cultivar. Pois, o mau uso modifica o dano. Os fatores que determinam a qualidade do solo, são essencialmente aquelas propriedades que têm grande influência no desenvolvimento das raízes, como agregação, retenção de água, teores de nutrientes, presença de patógenos, biomassa microbiana, etc. Por isso, o manejo do solo é uma das práticas mais importantes no sistema orgânico de cultivo. Segundo Gaidashova et al. (2009), a altitude também tem grande influência no volume radicular que pode interferir no nível de dano por nematoides. Entendemos que, porquanto o organomineral não fO efeito de práticas culturais, como a cobertura do solo mais densa, além de favorecer o vigor das plantas, mantêm bom nível de fertilidade dos solos e favorece a uniformidade das plantas (GAIDASHOVA et al., 2009).

Os índices das comunidades de nematoides do solo podem constituir importante ferramenta para detectar os diferentes tipos de manejo. De acordo com Ritzinger et al. (2008), a redução ou aumento da população de nematoides de vida livre, bem como a de fitonematoides dependeu da variedade utilizada, da população do nematoide no solo e do tratamento orgânico para a cultura da bananeira sob diferentes tipos de cobertura vegetal. Trabalhos desenvolvidos em Uganda, em bananais com utilização de fertilização e cobertura vegetal, revelam que o sistema pode ser rentável e pagar os custos de aplicação com o aumento da produção. Entretanto, não se pode generalizar a recomendação, devido não somente à variabilidade regional, mas também à resposta de cada cultivar ao fertilizante, bem como o seu custo (WAIGREGI;ASTEN, 2010). Neste trabalho, constatou-se que, na área 1, na presença do organomineral, o $\mathrm{pH}$ do solo foi mais elevado, como também o teor de $\mathrm{Ca}$ e o valor da saturação por bases $(\mathrm{V} \%)$. O aumento do teor de matéria orgânica foi insignificante $(0,06 \%)$. Assim, não houve contribuição do organomineral nos atributos químicos do solo, considerando que ambas as áreas apresentam saturação por bases acima de $70 \%$ (recomendada para a bananeira), altos teores de $\mathrm{K}$ (acima de $0,60 \mathrm{cmolc} /$ $\mathrm{dm} 3$ ) e baixos teores de $\mathrm{P}$ (menor que $6 \mathrm{mg} / \mathrm{dm} 3$ ) e baixos teores de matéria orgânica $(7,1 \mathrm{a} 20 \mathrm{~g} / \mathrm{kg})$.

Enquanto na área 2, constatou-se maior $\mathrm{pH}$, maiores teores de $\mathrm{K}, \mathrm{Ca}$ e $\mathrm{Mg}$; desta forma, maior valor de SB (soma das bases), CTC e saturação por bases $(\mathrm{V} \%)$. O teor de matéria orgânica, apesar de baixo, é $16 \%$ superior ao da área sem organomineral. Por outro lado, o teor de P é significativamente mais elevado na área sem organomineral. Contudo, por ser um solo mais arenoso, observou-se um efeito positivo do organomineral nas bases do solo $(\mathrm{K}, \mathrm{Ca}$ e $\mathrm{Mg}$ ).

$\mathrm{Na}$ área 3, não foi possível efetuar a comparação sem a aplicação de organomineral. Contudo, o solo apresenta apenas baixo teor de $\mathrm{P}$, estando os demais atributos adequados para a cultura da banana. 


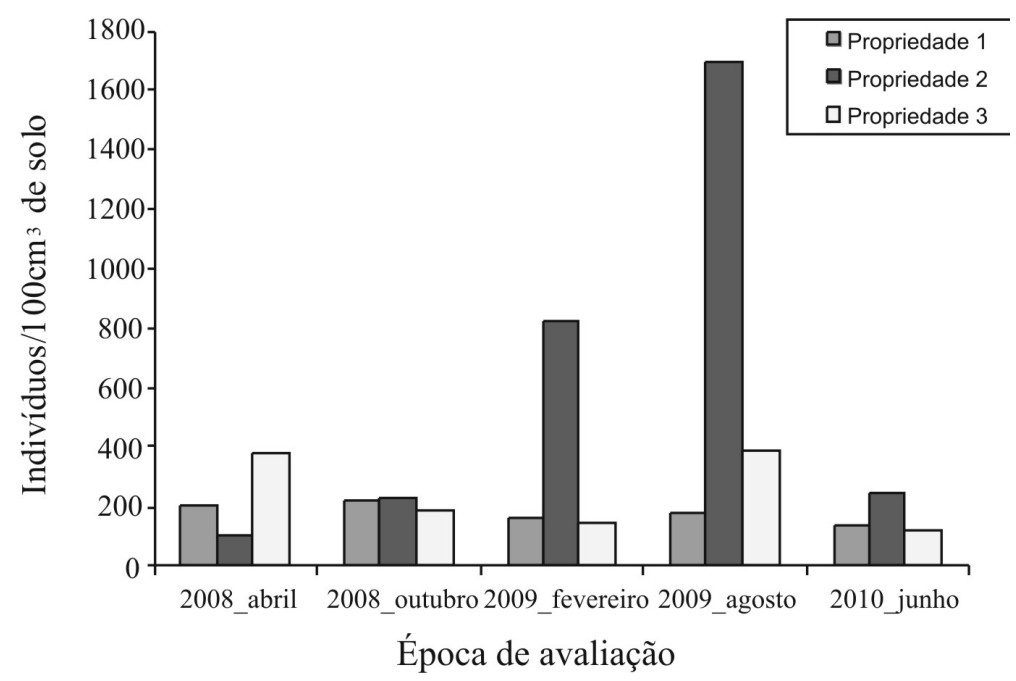

FIGURA 1- Número de indivíduos de Meloidogyne spp. nas amostras de solo extraídas desde 2008 até 2010, nos três diferentes cultivos de banana cv. Williams estudados. Bom Jesus da Lapa-BA. $2008-2010$.

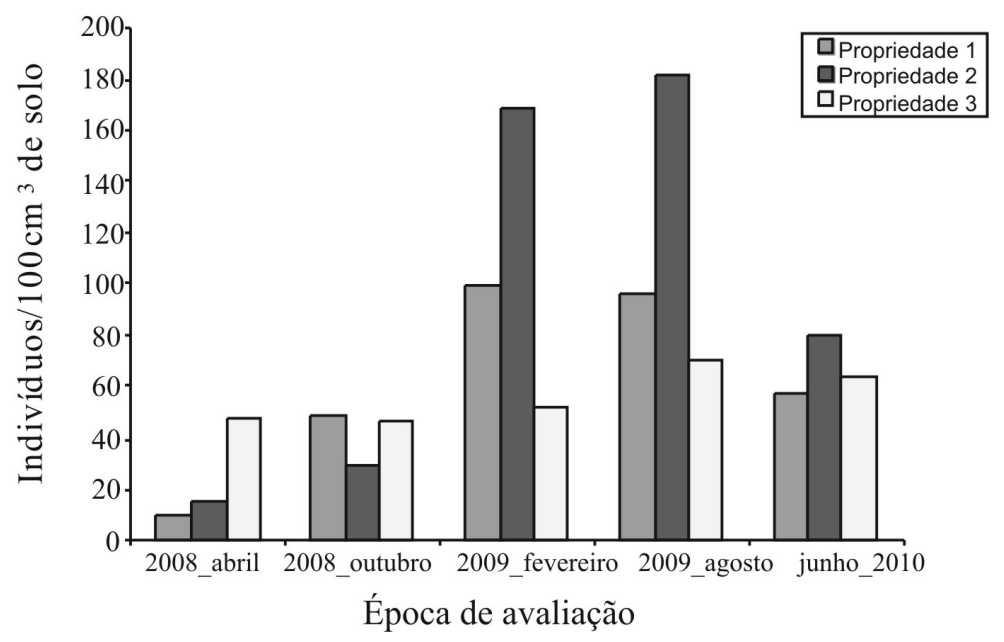

FIGURA 2- População de nematoides de vida livre (indivíduos $/ 100 \mathrm{~cm}^{3}$ de solo) nas amostras de solo extraídas desde 2008 até 2010, nos três diferentes cultivos de banana cv. Williams estudados. Bom Jesus da Lapa-BA. 2008 - 2010. 


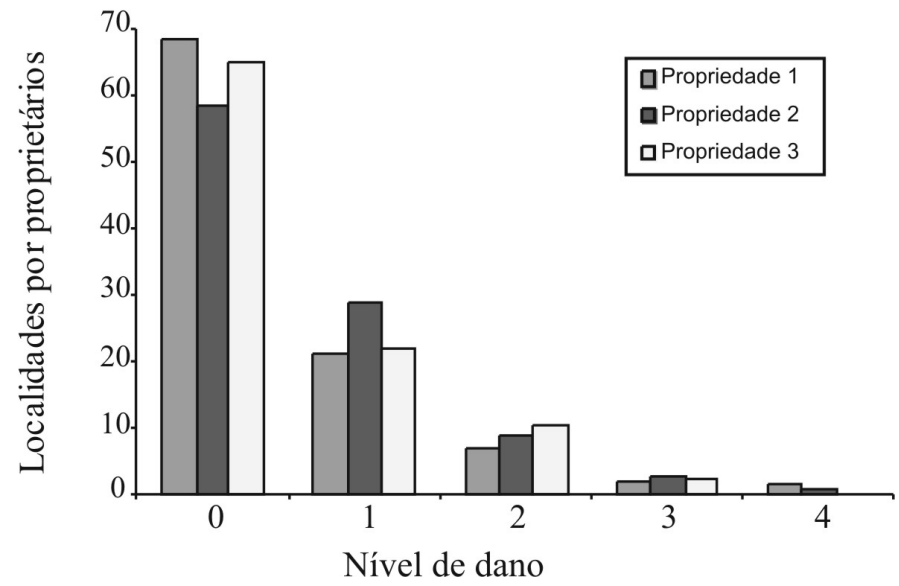

FIGURA 3 - Nível de dano utilizando-se de escala visual (Escala Bridge e Gowen,1993), conforme descrita em metodologias para monitoramento por Cordeiro e Fancelli (2008). Onde 0, representa nenhum dano; 1 , de 1 a $25 \%$; 2, de 26 a $50 \%$; 3 , de 51 a $75 \%$, e 4 dano acima de $76 \%$.

TABELA 1 - Efeito interativo dos fatores avaliados nos três diferentes cultivos de banana cv. Williams, nos anos de 2008, 2009 e 2010. Bom Jesus da Lapa-BA. 2008 - 2010.

\begin{tabular}{lcc}
\hline & \multicolumn{2}{c}{ Quadrados médios } \\
\hline Fonte de variação & Meloidogyne spp. & Nematoide de vida livre \\
\hline Ano & $0,0001 * *$ & $0,0001 * *$ \\
Organomineral & $0,5944 \mathrm{~ns}$ & $0,0687 \mathrm{~ns}$ \\
Propriedade & $0,0001 * *$ & $0,1150 \mathrm{~ns}$ \\
Ano x organomineral & $0,4378 \mathrm{~ns}$ & $0,8684 \mathrm{~ns}$ \\
Ano x propriedade & $0,0001 * *$ & $0,0023 * *$ \\
Organomineral x propriedade & $0,026^{*}$ & $0,3173 \mathrm{~ns}$ \\
Ano x organomineral x propriedade & $0,1865 \mathrm{~ns}$ & $0,5049 \mathrm{~ns}$ \\
\hline CV $(\%)$ & 18,17 & 19,78 \\
\hline
\end{tabular}

*, **, ns: Significativo a $5 \%, 1 \%$ e não significativo, respectivamente.

TABELA 2 - Número de indivíduos de nematoides nas amostras de solo extraídas desde 2008 até 2010, nos três diferentes cultivos de banana cv. Williams avaliados. Bom Jesus da Lapa-BA. 2008 $-2010$.

\begin{tabular}{cccccc}
\hline \multicolumn{7}{c}{ Meloidogyne spp. (indivíduos/100 $\mathrm{cm}^{3}$ de solo) } \\
\hline Local & Abril/2008 & Outubro/2008 & Fevereiro/2009 & Agosto/2009 & Junho/2010 \\
\hline Propriedade 1 & 204,4 ab A & 220,0 a A & 159,7 b A & 186,2 c A & 141,7 a A \\
Propriedade 2 & 102,8 b B & 229,7 a B & 827,7 a A & 1692,0 a A & 245,5 a B \\
Propriedade 3 & 379,3 a A & 189,4 a AB & $146,9 \mathrm{~b} \mathrm{~B}$ & 389,4 b A & 121,2 a B \\
\hline \multicolumn{7}{c}{ Nematoides de vida livre (indivíduos/100/cm3 de solo) } \\
Propriedade 1 & $10,3 \mathrm{~b} \mathrm{~B}$ & 48,4 a A & 99,2 ab A & 96,2 a A & 57,0 a A \\
Propriedade 2 & 15,6 ab B & 29,4 a B & 168,7 a A & 181,7 a A & 79,5 a A \\
Propriedade 3 & 47,1 a A & 46,9 a A & 51,9 b A & 70,0 a A & 64,4 a A \\
\hline
\end{tabular}

Médias seguidas pela mesma letra minúscula na coluna e pela mesma letra maiúscula na linha não diferem significativamente entre si, pelo teste-t. 
TABELA 3 - Efeito do organomineral sobre a população de Meloidogyne nos três diferentes cultivos de banana cv. Williams avaliados. Bom Jesus da Lapa-BA. 2008 - 2010.

\begin{tabular}{lcc}
\hline & \multicolumn{2}{c}{ População de Meloidogyne spp. $\left(100 \mathrm{~cm}^{3}\right.$ de solo) } \\
\cline { 2 - 3 } & Com organomineral & Sem organomineral \\
\hline Propriedade 1 & $200,4 \mathrm{~A} \mathrm{~b}$ & $159,2 \mathrm{Ab}$ \\
Propriedade 2 & $618,8 \mathrm{Aa}$ & $699,1 \mathrm{Aa}$ \\
Propriedade 3 & $241,8 \mathrm{ab}$ & - \\
\hline
\end{tabular}

Médias seguidas pela mesma letra minúscula nas colunas e maiúscula na linha não diferem estatisticamente entre si, pelo teste de Tukey, a $5 \%$.

TABELA 4 - Correlações entre nível de dano e população de Meloidogyne spp. com e sem a utilização do produto organomineral, nos três diferentes cultivos de banana cv. Williams avaliados. Bom Jesus da Lapa-BA. 2008 - 2010.

\begin{tabular}{|c|c|c|c|c|c|c|c|c|c|c|}
\hline \multirow{3}{*}{ Propriedade 1} & \multicolumn{5}{|c|}{ Sem organomineral } & \multicolumn{5}{|c|}{ Com organomineral } \\
\hline & \multicolumn{5}{|c|}{ Nível de dano } & \multicolumn{5}{|c|}{ Nível de dano } \\
\hline & 0 & 1 & 2 & 3 & 4 & 0 & 1 & 2 & 3 & 4 \\
\hline & 0.238 & -0.320 & 0.024 & -0.133 & & 0.153 & -0.263 & 0.030 & -0.188 & -0.144 \\
\hline & ns & ns & ns & $\mathrm{ns}$ & - & ns & $\mathrm{ns}$ & ns & $\mathrm{ns}$ & ns \\
\hline \multirow{4}{*}{ Propriedade 2} & \multicolumn{5}{|c|}{ Nível de dano } & \multicolumn{5}{|c|}{ Nível de dano } \\
\hline & 0 & 1 & 2 & 3 & 4 & 0 & 1 & 2 & 3 & 4 \\
\hline & 0.119 & 0.166 & -0.198 & -0.096 & 0.108 & -0.156 & -0.383 & -0.229 & -0.374 & -0.124 \\
\hline & ns & ns & ns & ns & ns & ns & $*$ & ns & $*$ & ns \\
\hline \multirow{3}{*}{ Propriedade $3^{-}$} & \multicolumn{5}{|c|}{ Nível de dano } & \multicolumn{5}{|c|}{ Nível de dano } \\
\hline & 0 & 1 & 2 & 3 & 4 & 0 & 1 & 2 & 3 & 4 \\
\hline & $\begin{array}{c}-0.197 \\
n s\end{array}$ & $\begin{array}{c}-0.103 \\
n s\end{array}$ & $\begin{array}{c}-0.083 \\
n s\end{array}$ & $\begin{array}{c}0.111 \\
n s\end{array}$ & - & & & & & \\
\hline
\end{tabular}

${ }^{1}$ - Níveis de danos (Escala Bridge \& Gowen,1993), conforme descrito em metodologias para monitoramento por Cordeiro e Fancelli (2008).

TABELA 5- Efeito da utilização do produto organomineral no percentual de nível de dano promovido por nematoides nos três diferentes cultivos de banana cv. Williams. Bom Jesus da Lapa-BA, 2010

\begin{tabular}{|c|c|c|c|c|c|c|c|c|c|c|}
\hline \multirow{3}{*}{ Propriedade 1} & \multicolumn{5}{|c|}{ Sem organomineral } & \multicolumn{5}{|c|}{ Com organomineral } \\
\hline & \multicolumn{5}{|c|}{ Nível de dano } & \multicolumn{5}{|c|}{ Nível de dano } \\
\hline & 0 & 1 & 2 & 3 & 4 & 0 & 1 & 2 & 3 & 4 \\
\hline & $71,0 \mathrm{a}$ & $20,8 \mathrm{a}$ & $6,8 \mathrm{a}$ & $1,4 \mathrm{a}$ & $0,0 \mathrm{~b}$ & $66,0 \mathrm{a}$ & $21,0 \mathrm{a}$ & $7,5 \mathrm{a}$ & $2,5 \mathrm{a}$ & $3,1 \mathrm{a}$ \\
\hline \multirow{3}{*}{ Propriedade 2} & \multicolumn{5}{|c|}{ Nível de dano } & \multicolumn{5}{|c|}{ Nível de dano } \\
\hline & 0 & 1 & 2 & 3 & 4 & 0 & 1 & 2 & 3 & 4 \\
\hline & $59,9 \mathrm{a}$ & $29,4 \mathrm{a}$ & $7,2 \mathrm{a}$ & $2,4 a$ & $1,1 \mathrm{a}$ & $57,6 \mathrm{a}$ & $28,8 \mathrm{a}$ & $10,2 \mathrm{a}$ & $3,1 \mathrm{a}$ & $0,4 a$ \\
\hline \multirow{3}{*}{ Propriedade 3} & \multicolumn{5}{|c|}{ Nível de dano } & \multicolumn{5}{|c|}{ Nível de dano } \\
\hline & 0 & 1 & 2 & 3 & 4 & 0 & 1 & 2 & 3 & 4 \\
\hline & - & - & - & - & - & 65,0 & 22,1 & 10,7 & 2,1 & 0,0 \\
\hline
\end{tabular}

Médias seguidas pela mesma letra nas colunas não diferem estatisticamente entre si, pelo teste de Tukey, a $5 \%$ de probabilidade. ${ }^{1}$ - Níveis de danos (Escala Bridge e Gowen,1993), conforme descrito em metodologias para monitoramento por Cordeiro e Fancelli (2008). 


\section{CONCLUSÕES}

1- Depois de três anos de observação, com o uso ou não do organomineral, as populações de nematoides de vida livre aumentam nas bananeiras Williams cultivadas em Bom Jesus da Lapa-BA, demonstrando que este produto não possui efeito nematicida.

2- Os nematoides Meloidogyne incognita e $M$. javanica são as espécies mais ocorrentes nas bananeiras Williams cultivadas em Bom Jesus da Lapa-BA.

3- O nível de dano promovido pelos nematoides e baixo, não podendo ser explicado pelo uso do organomineral.

4- O monitoramento é essencial para embasar a necessidade de aplicação do organomineral e/ ou de produtos químicos.

5- O manejo cultural interfere no desenvolvimento da população de fitonematoides e de nematoides de vida livre.

\section{AGRADECIMENTOS}

Uganda. Agricultural Systems 2010 (no prelo).

\section{REFERÊNCIAS}

BORGES, A. L.; SOUZA, L. da S. Atributos físicos e químicos de solos cultivados com bananeira, sob irrigação, no Projeto Formoso. Cruz das Almas: Embrapa Mandioca e Fruticultura Tropical, 2009. 33 p. (Boletim de Pesquisa e Desenvolvimento, 42).

CORDEIRO, Z.J.M.; BORGES, A.L.; FANCELLI, M. et al. Produção integrada de banana. In: Brasil. Ministério da Agricultura, Pecuária e Abastecimento. Produção integrada no Brasil: agropecuária sustentável alimentos seguros. Brasília: Mapa; ACS, 2009. p.238-260.

CORDEIRO, Z.J.M.; FANCELLI, M. (Ed.). Produção integrada de banana: metodologias para monitoramentos. Cruz das Almas: Embrapa Mandioca e Fruticultura Tropical, 2008. 52 p. (Documentos, 175).
GAIDASHOVA, S. V.; VAN ASTEN, P.; DE WAELE, D. et al. Relationship between soil properties, crop management, plant growth and vigour, nematode occurrence and root damage in East African Highland banana-cropping systems: a case study in Rwanda. Nematology, Leiden, v.11, n.6, p.883-894, 2010.

HARTMAN, J.B.; VUYLSTEKE, D.; SPEIJER, P.R. et al. De. Measurement of the field response of Musa genotypes to Radopholus similis and Helicotylenchus multicinctus and the implications for nematode resistance breeding. Euphytica, Wageningen, v.172, p.139-148, 2010.

JENKINS, W.R. A rapid centrifugal-flotation technique for separating nematodes from soil. Plant Disease Reporter,Washington, v.48, p.692, 1964.

PORTOCARRERO, M. A.; KOSOSKI, A. R. Alimentos seguros: uma política de governo. In: BRASIL. Ministério da Agricultura, Pecuária e Abastecimento. Produção integrada no Brasil: agropecuária sustentável alimentos seguros. Brasília: Mapa; 2009. p. 13-29.

RITZINGER, C.H.S.P.; FANCELLI, M.; CORDEIRO, Z.J.M. et al. Nematoides em bananeira em áreas de produção integrada da cultura, com utilização de fertilizante organomineral, no Projeto de Irrigação de Formoso, em Bom Jesus da Lapa-BA. In: ZAMBOLIM, L.; NASSER, L.C.B.; ANDRIGUETO, J.R.; TEIXEIRA, J.M.; FACHINELLO, J.C. (Org.). Produção integrada no Brasil. Brasília: CNPq; Viçosa: Universidade Federal de Viçosa, 2008.

RITZINGER, C.H.S.P.; FANCELLI, M.; BORGES, A.L. et al. Nematoides em solo sob cultivo de bananeira 'Prata-Anã' e 'Caipira' sob diferentes tipos de manejo orgânico. In: CONGRESSO BRASILEIRO DE FRUTICULTURA, 20. ANNUALMEETINGOFTHEINTERAMERICAN SOCIETY FOR TROPICAL HORTICULTURE, 54., Vitória, 2008. Anais...

WAIREGI, L.W.I.;VAN ASTEN, P.J.A. The agronomic and economic benefits of fertilizer and mulch use in highland banana systems in Uganda. Agricultural Systems, Essex, v.103, n.8, p.543-550, 2010 